This item was submitted to Loughborough's Research Repository by the author.

Items in Figshare are protected by copyright, with all rights reserved, unless otherwise indicated.

\title{
Noninvasive imaging of signals in digital circuits
}

PLEASE CITE THE PUBLISHED VERSION

http://dx.doi.org/10.1063/1.1447302

PUBLISHER

(C) American Institute of Physics

VERSION

VoR (Version of Record)

LICENCE

CC BY-NC-ND 4.0

REPOSITORY RECORD

Gebrial, W., R.J. Prance, T.D. Clark, C.J. Harland, H. Prance, and Mark J. Everitt. 2019. "Noninvasive Imaging of Signals in Digital Circuits". figshare. https://hdl.handle.net/2134/12071. 
This item was submitted to Loughborough's Institutional Repository (https://dspace.lboro.ac.uk/) by the author and is made available under the following Creative Commons Licence conditions.

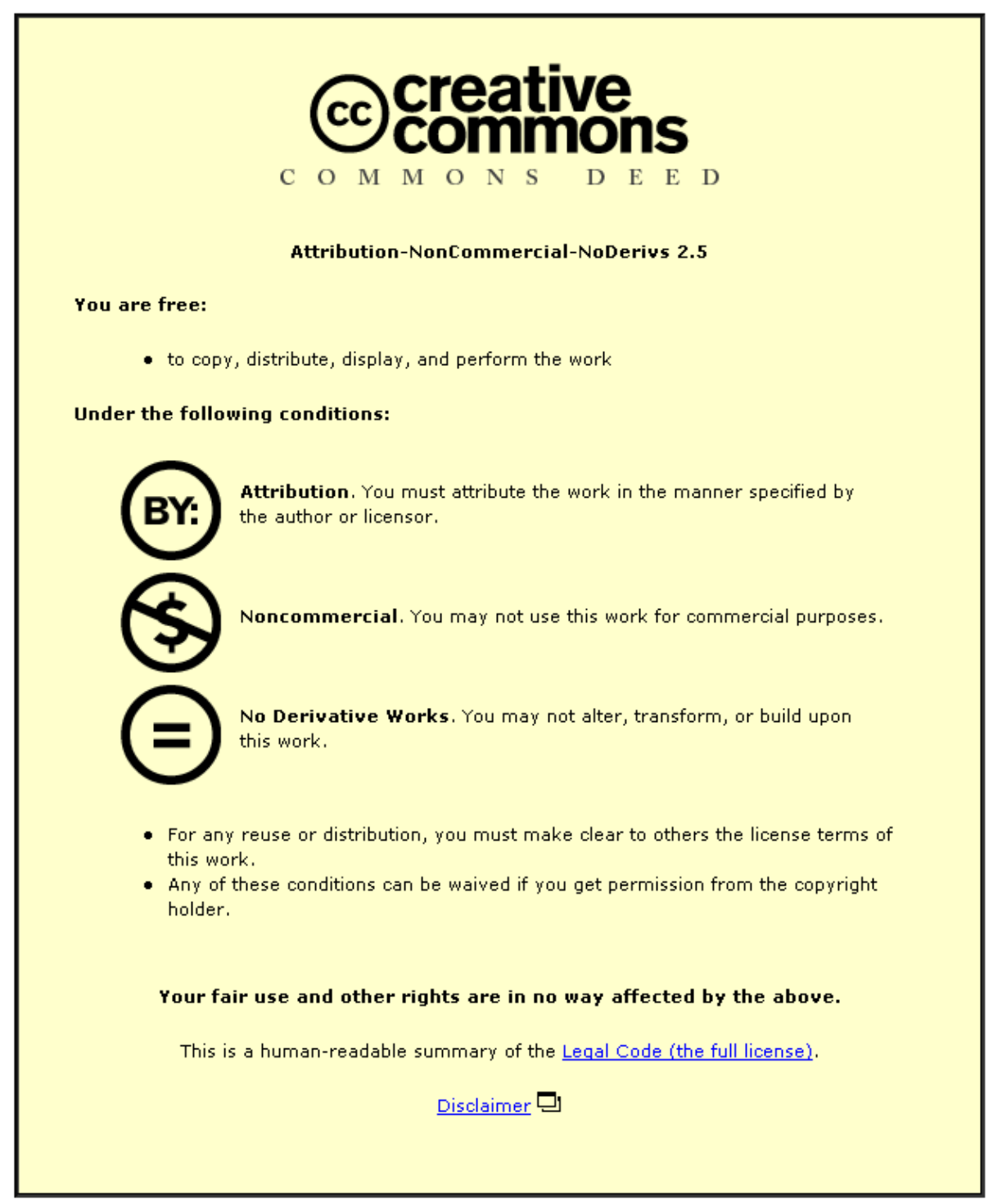

For the full text of this licence, please go to: http://creativecommons.org/licenses/by-nc-nd/2.5/ 


\section{AIP gations Scientific Instruments}

\section{Noninvasive imaging of signals in digital circuits}

W. Gebrial, R. J. Prance, T. D. Clark, C. J. Harland, H. Prance et al.

Citation: Rev. Sci. Instrum. 73, 1293 (2002); doi: 10.1063/1.1447302

View online: http://dx.doi.org/10.1063/1.1447302

View Table of Contents: http://rsi.aip.org/resource/1/RSINAK/v73/i3

Published by the American Institute of Physics.

Additional information on Rev. Sci. Instrum.

Journal Homepage: http://rsi.aip.org

Journal Information: http://rsi.aip.org/about/about_the_journal

Top downloads: http://rsi.aip.org/features/most_downloaded

Information for Authors: http://rsi.aip.org/authors 


\title{
Noninvasive imaging of signals in digital circuits
}

\author{
W. Gebrial, R. J. Prance, T. D. Clark, ${ }^{a)}$ C. J. Harland, H. Prance, and M. Everitt \\ Centre for Physical Electronics, School of Engineering, University of Sussex, Brighton, \\ Sussex BN1 9QT, United Kingdom
}

(Received 24 July 2001; accepted for publication 29 October 2001)

\begin{abstract}
In this article we describe the construction and use of a noninvasive (noncontact) electric potential probe to measure time delays of signals propagating through digital circuits. As we show, by incorporating such probes into a scanning microscope system we have been able to create time delay images of these signals. We suggest that future developments of this technique may lead to real time, high resolution imaging of digital pulses across complex very large scale integrated circuits. (C) 2002 American Institute of Physics. [DOI: 10.1063/1.1447302]
\end{abstract}

\section{INTRODUCTION}

In the design of a high speed digital system based on integrated circuit (IC) chips it is clearly of great importance to establish the propagation time delays of signal pulses at various points in the system. In principle, the data sheets provided by manufacturers contain the time delay information for any specified IC. However, this information relates only to the chip itself (or rather when it is embedded in a particular measurement circuit). Actual timings may be very different when such ICs are built into real digital systems. Other factors, such as those arising from interconnections, will contribute significantly to overall propagation time delays. These problems tend to be compounded when digital ICs are run close to their maximum input frequency. In this situation maintenance of correct timing can be difficult to achieve. Clearly, it would be of great advantage to be able to measure propagation delays noninvasively in situ in real digital circuit systems. This provides the motivation behind the work described in the article.

In an earlier article $^{1}$ we described the development of ultrahigh input impedance electric potential sensors based on standard commercial operational amplifiers. The principal feature of these sensors is the electronically enhanced input impedance. This enhancement is achieved by the use of a variety of feedback techniques leading to input impedances as high as $10^{17} \Omega$ at $1 \mathrm{~Hz}$. These sensors can then function as (almost) perfect voltmeters for ac signals from various sources provided the input impedance is much larger than the sensor-source impedance. Given such high impedances it has proved possible to detect electric potentials (referred to a standard potential—usually earth) without making direct electrical contact to the source of these potentials. Furthermore, the sensors operate on displacement, not real charge. Hence, these sensors can be used noninvasively, with no possibility of mechanical or (to all intents and purposes) electrical damage to any circuit under test. With such capabilities the sensors have already been used in a range of noninvasive applications including the remote monitoring of

\footnotetext{
${ }^{a}$ Author to whom correspondence should be addressed; electronic mail: t.d.clark@sussex.ac.uk
}

electrocardiograms, ${ }^{1}$ noncontact dielectric measurements, ${ }^{2}$ and very large scale integration circuit imaging. ${ }^{3}$ In these applications the electric potential sensors have clear advantages over other existing techniques ${ }^{4-8}$ since they provide direct information concerning the behavior of sources of interest without any dependency on physical models of these sources. In particular, we are able to monitor signals and measure voltage and phase remotely. In the most recent published version ${ }^{9}$ we achieved noise floors of $2 \mu \mathrm{V} / \sqrt{\mathrm{Hz}}$ at 1 $\mathrm{Hz}$ down to $0.1 \mu \mathrm{V} / \sqrt{\mathrm{Hz}}$ at $1 \mathrm{kHz}$ referred to the input. This has now been improved at optimal coupling to $70 \mathrm{nV} / \sqrt{\mathrm{Hz}}$ at $1 \mathrm{~Hz}$ referred to the input. In each case the bandwidth was restricted to the range $0.01 \mathrm{~Hz}-100 \mathrm{kHz}$ due to limitations in the operational amplifiers used (e.g., the INA 116UA). This is obviously quite insufficient for probing high frequency digital circuits. However, from a circuit viewpoint we are not constrained to just low frequency opamps. There exist several commercial opamps with a sufficiently high gain bandwidth product to be used in monitoring and imaging pulse propagation in a range of digital circuits.

In practice two general techniques are used to measure propagation time delays in digital circuits. In the first (and main) method the phase difference is measured between the input to the circuit and its output, i.e., the phase angle between the input (rectangular) pulse and the output is found by measuring the time difference between two zero crossing points. For digital circuits operating at tens of $\mathrm{MHz}$ this time interval may be in the nanosecond range. In the second method heterodyning techniques are used to mix down from a high operating frequency (with concomitantly small time intervals to measure) to a lower frequency where this time interval, being stretched, is easier to measure. Conventionally, both methods require direct electrical contact be made to the digital circuit. However, by substituting an ultrahigh impedance electric potential sensor for a direct contact probe, a new approach can be adopted for these measurement techniques. As we shall demonstrate, the combination of a suitably designed sensor, and a computer controlled threeaxis $(x, y, z)$ scanning system, has allowed us to follow noninvasively pulses moving through a variety of digital circuits, at repetition frequencies of up to $10 \mathrm{MHz}$. With the technique established, there is every possibility that this fre- 


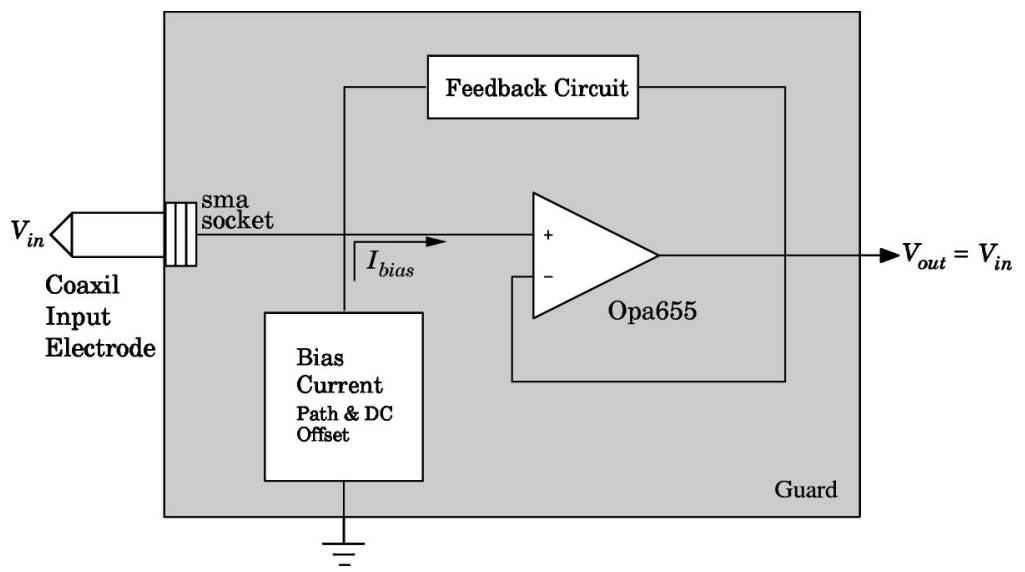

FIG. 1. Schematic diagram of the high impedance electric potential probe based on the OPA $655 \mathrm{P}$ operational amplifier. quency range can be extended into the $100 \mathrm{MHz}$ range and beyond. We also note that this technique for following electronic pulses could have more widespread application. Thus, the in vitro (and in saline) noninvasive detection of signals propagating through, for example, muscle or neuronal tissue looks distinctly possible in the future.

\section{THE HIGH FREQUENCY ELECTRIC POTENTIAL SENSOR}

In our earlier work on electric potential sensors we were able to enhance very substantially the dynamical (i.e., ac) input impedance in various electrometer grade operational amplifiers. Initially our designs were based on the OPA $111 \mathrm{AM}^{10}$ followed by the INA $116 \mathrm{UA} .^{11}$ To achieve our design goals the sensor was configured as a stable buffer amplifier with precisely unity gain. Various guarding, feedback, and biasing circuit techniques were added to boost input impedance and maintain stable operation. Although very successful in the audio range, we now had the problem of monitoring high speed digital pulses. Specifically, we needed to incorporate an opamp with a very much higher unity gain bandwidth than is available using either the OPA 111 or the INA 116. One suitable opamp is the Burr Brown OPA $655 \mathrm{P}^{10}$ which has a $400 \mathrm{MHz}$ unity gain bandwidth, and a low input bias current requirement (5 pA). From the specifications provided by the manufacturer, this opamp has a dc resistance of $10^{12} \Omega$ in parallel with an input capacitance of $1 \mathrm{pF}$.

In practice, in developing the OPA 655P based sensor we adopted essentially the same design as we used previously in the audio frequency versions. Thus, we utilized the same guarding and feedback techniques to enhance input impedance. ${ }^{3}$ In Fig. 1 we show a block diagram of the OPA $655 \mathrm{P}$ sensor. In this design we paid close attention to the

TABLE I. Input impedance of OPA 655P without feedback and with feedback.

\begin{tabular}{lcc}
\hline \hline $\begin{array}{l}\text { Frequency } \\
\mathrm{MHz}\end{array}$ & Without feedback & With feedback \\
\hline 1 & $0.16 \mathrm{M} \Omega$ & $1.9 \mathrm{M} \Omega$ \\
4 & $0.04 \mathrm{M} \Omega$ & $0.48 \mathrm{M} \Omega$ \\
8 & $0.02 \mathrm{M} \Omega$ & $0.24 \mathrm{M} \Omega$ \\
10 & $0.016 \mathrm{M} \Omega$ & $0.20 \mathrm{M} \Omega$ \\
\hline \hline
\end{tabular}

physical arrangement of the guard so as to reduce to a minimum any stray capacitance at the input of the sensor, an essential step in maximizing the input impedance of the probe. To maintain a flat frequency response even with an increasing capacitive load, a small $27 \Omega$ isolation resistor was built into the output of the sensor.

We determined the input impedance of the sensor at a particular frequency by varying the coupling capacitance to a source of known (and fixed) voltage until the output voltage of the sensor (at unity gain) was reduced by a factor of 2 . At this setting the coupling and probe input capacitances were equal, allowing us to calculate the capacitive reactance of the probe. In Table I we show a plot of the measured input impedance of the OPA 655P sensor at spot frequencies between 1 and $10 \mathrm{MHz}$. In Fig. 2 we show the measured gain response of this sensor between $10 \mathrm{kHz}$ and $500 \mathrm{MHz}$ with the sensor coupled to a fixed voltage source through a coupling capacitor of $6.8 \mathrm{pF}$. As can be seen in this figure, unity gain is maintained between $100 \mathrm{kHz}$ and $100 \mathrm{MHz}$. This was quite sufficient for our purposes, allowing us to follow pulses up to a repetition frequency of $10 \mathrm{MHz}$ with good edge discrimination. As an example of the performance of the sensor, we show in Fig. 3 the open circuit noise spectral density plot over the frequency range $1 \mathrm{MHz}-10 \mathrm{MHz}$.

The spatial resolution of the sensor was set by the 0.025 $\mathrm{mm}$ radius of curvature of its probe tip (the pointed inner of

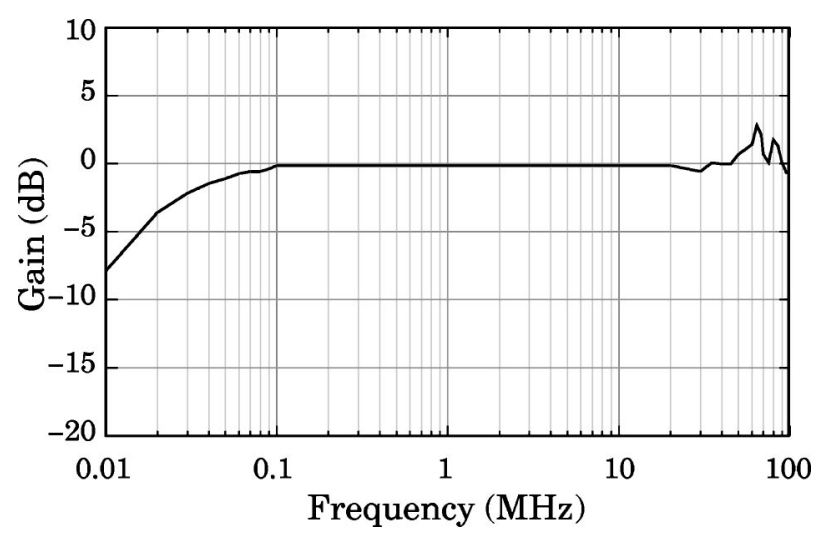

FIG. 2. Measured gain response versus frequency for the OPA 655 based sensor probe using a source-probe coupling capacitance of $6.8 \mathrm{pF}$. Here, a precise unity gain response is maintained between $100 \mathrm{kHz}$ and $100 \mathrm{MHz}$. The output was coupled through two parallel capacitors of $0.1 \mu \mathrm{F}$ and $33 \mathrm{pF}$. 


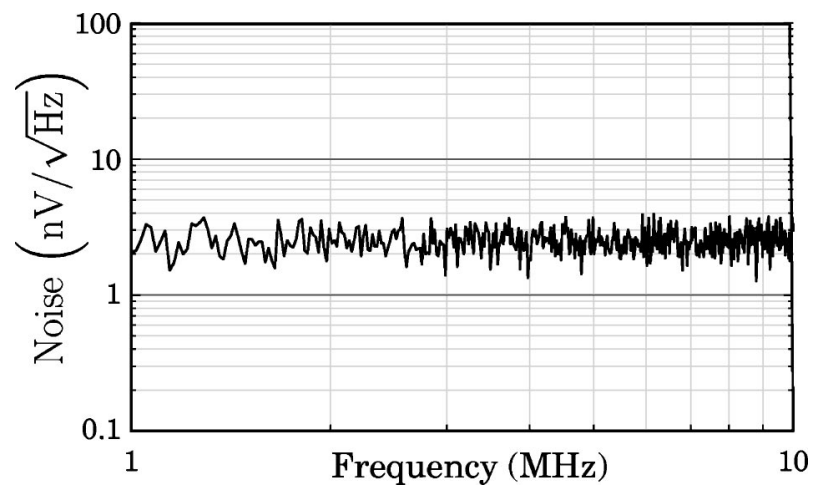

FIG. 3. Open circuit noise response of the OPA 655P between 1 and 10 $\mathrm{MHz}$ referred to the input.

the coaxial input depicted in Fig. 1). In the circuits discussed in this article the resolution required was, in fact, moderate ( $0.2 \mathrm{~mm}$ step length between measurements) and the radius of curvature criterion could be met quite adequately using UT85 coaxial cable with a mechanically sharpened and polished inner conductor. However, as we have demonstrated previously, ${ }^{3}$ this coaxial cable approach can easily be scaled down to provide spatial resolutions at the $\mu \mathrm{m}$ level. ${ }^{12}$

\section{THE SCANNING IMAGING SYSTEM}

The high frequency sensor described in Sec. II was used in conjunction with a scanning probe translation system. For our work the sample (i.e., a digital circuit) was mounted on a three-axis $(x, y, z)$ linear movement stage while the sensor was maintained at a fixed position above this stage. In operation the stage plus sample circuits, maintained at a fixed distance $(z)$ below the sensor, was moved in discrete steps along either the $x$ or $y$ directions, typically in a raster pattern. At each discrete position of the stage we took a reading of the electric potential at the sensor probe tip. This allowed us to build up an $x-y$ plane data array for the potential at height $z$ above the circuit. The data set could then be converted, via a computer interface and suitable software, into an electrical image of the circuit. For the commercial stage system we used, ${ }^{13}$ the minimum translations in the $x-y$ plane and $z$ directions were 0.5 and $0.05 \mu \mathrm{m}$, respectively, more than sufficient for our needs. The sample circuit was mounted on a commercial gimballed positioning system below the sensor probe. This was used to adjust the surface of the sample circuit so that it was parallel to the movement of the stage in the $x-y$ plane. The position and the scanning translational movements in each of the two orthogonal $(x, y)$ directions were controlled by digital positioning system display controller through a virtual instrumentation program created under National Instruments LABVIEW software. ${ }^{14}$ This could also be used to change the $z$ axis separation between the surface of the sample circuit and the sensor probe. Further programs were generated under LABVIEW to take care of the data collection and processing from which propagation time delay circuit images were created.

With the scanning-probe system in operation, we made use of two different methods to measure propagation time delays. In the first method pulse wave forms were displayed

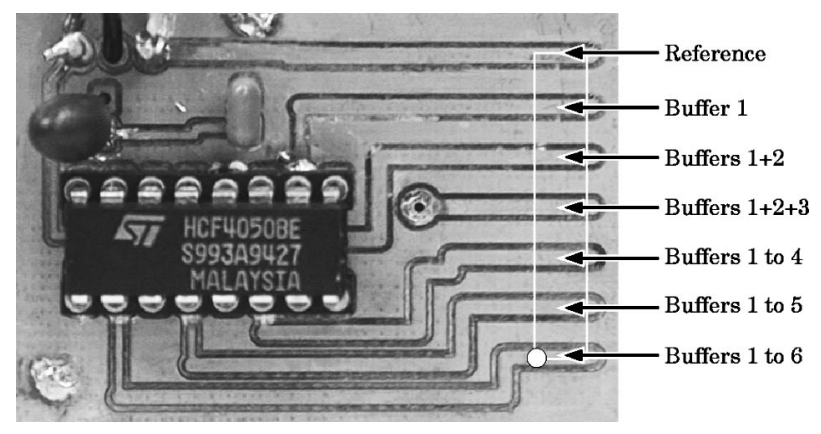

FIG. 4. Photograph of the noninverting Hex buffer HCF4050UBE with selected scan areas shown delineated by the rectangular box.

on a multichannel, digitizing oscilloscope-in this work a Tektronix TDS520 model. Through this display we could then compare the time delay between input channel 1 (the reference channel for the signal supplied to the sample circuit) and output channel 2 (for the pulse picked up by the sensor probe on the scanning system). In the second method the propagation time delay was measured directly by means of a very fast electronic counter-a Philips 6680 model with a $0.5 \mathrm{~ns}$ time resolution. In this instrument there is provision for external arming which enables the timing measurement to be triggered. In practice the counter measured the time delay between two channels A and B, where A is the reference and $\mathrm{B}$ is the signal pickup, and generated a number on its display which represented the delay. In order to prevent the counter from taking measurements when the sensor probe was not positioned over a track in the sample circuit, a comparator and a hysteresis circuit were used to provide an external trigger signal. This enabled us to start and stop the measurement automatically when positioned over a track, thereby preventing spurious readings from noise between tracks.

\section{EXPERIMENTAL RESULTS}

\section{A. Example 1: The HCF4050UBE complementary metal-oxide-semiconductor (CMOS) noninverting Hex buffer}

To illustrate the use of the scanning probe system to measure propagation time delays in digital pulse circuits we chose as our first example a Hex buffer-the HCF4050UBE ${ }^{15}$ — which is a monolithic IC with six noninverting buffers. A photograph of this circuit is shown in Fig. 4. The outputs of the six buffers were arranged so that precise (i.e., quantized) time delays could be added sequentially. Thus, the output of the first buffer was fed into the input of the second buffer, the output of the second buffer was then fed into the input of the third buffer, and so on. Using the probe system, we could then follow the increasing time delay (with reference to the input signal) as a pulse propagated, buffer by buffer, through the circuit. As can be seen in Fig. 4, there are seven delineated tracks on this PCB-mounted Hex buffer, one for the reference signal, and the other six for the sequence of buffer stages. The width of the tracks on the PCB was $1.5 \mathrm{~mm}$ and the spacing between adjacent tracks was $2 \mathrm{~mm}$. In our scanning runs the excess (remaining) metallization between adjacent tracks (Fig. 4) was connected to 

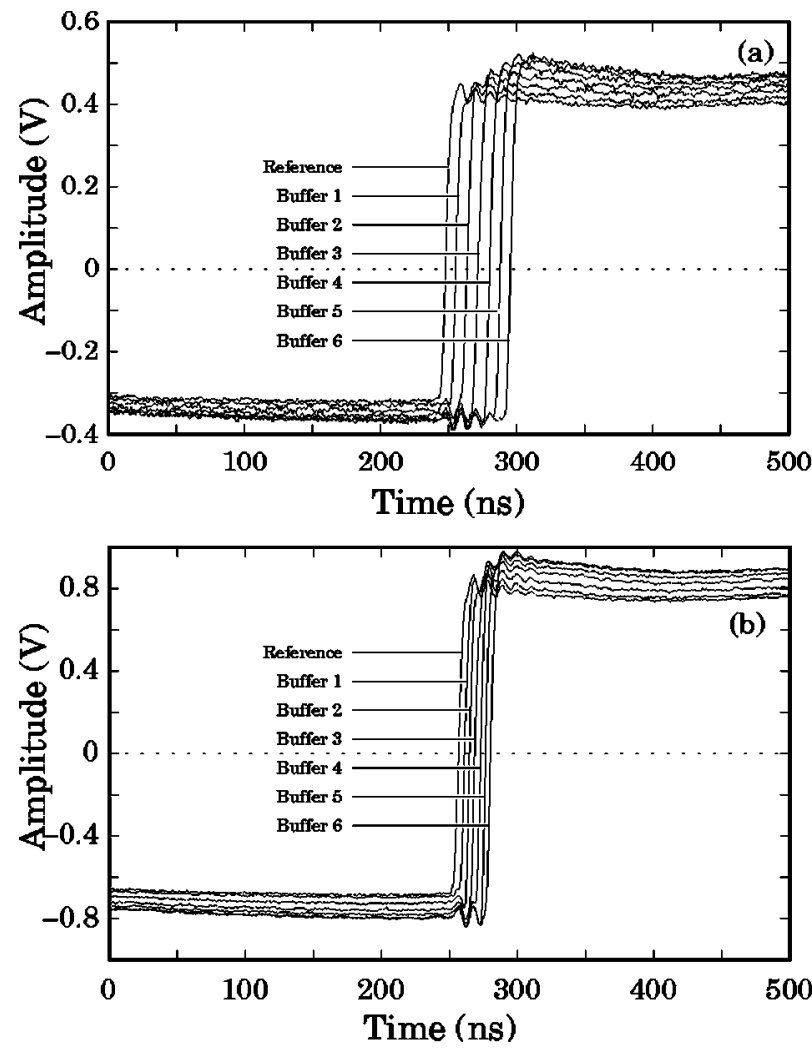

FIG. 5. Set of wave forms acquired using: (a) Tektronix TDS520 oscilloscope from all six buffers of the HCF4050UBE noninverting Hex buffer when supplied with $5 \mathrm{~V}$ and (b) Tektronix TDS520 oscilloscope from all six buffers of the HCF4050UBE noninverting Hex buffer when supplied with $10 \mathrm{~V}$.

ground. The $5 \mathrm{~V}$ (or $10 \mathrm{~V}$ ) supply for the Hex buffer was decoupled via 0.1 and $10 \mu \mathrm{F}$ capacitors. With the time delay resolution we had available ( $0.5 \mathrm{~ns})$, we certainly could not follow pulse propagation along the individual tracks on the board but we could resolve delays through each gate. Consequently, we chose to scan within the rectangular region ( $\Delta y=21.4 \mathrm{~mm}$ by $\Delta x=2.6 \mathrm{~mm}$ ) marked out in Fig. 4 since this enabled us to acquire timing information for each of these gates referred to the input signal. In our scanning runs, which were taken at a fixed vertical separation $(z)$ between the circuit and the sensor probe tip, the start point is denoted by the circle in Fig. 4.

As we have already stated, we had at our disposal two methods of following timing delays, either using a direct oscilloscope display or a very fast electronic counter. Our first approach was using the oscilloscope display. In Fig. 5(a) we show the time domain leading edges of seven pulses recorded on a Tektronix TDS520 digitizing oscilloscope. Reading left to right, the first is the reference and the last is the pulse after the sixth buffer stage. In this example the reference signal was a $1 \mathrm{MHz} / 5 \mathrm{~V}$ pulse which was connected to the input of the first buffer. The pulse shapes were recorded by making a line scan along the $y$ axis, at fixed $x$, across the rectangular area shown in Fig. 4 and at a probe height above the Hex buffer PCB of $15 \mu \mathrm{m}$. The scanning system was preset to stop in turn above each track on the buffer PCB. It is apparent from Fig. 5(a) that each of the pulse edges is well resolved and can be used to make time delay measurements.
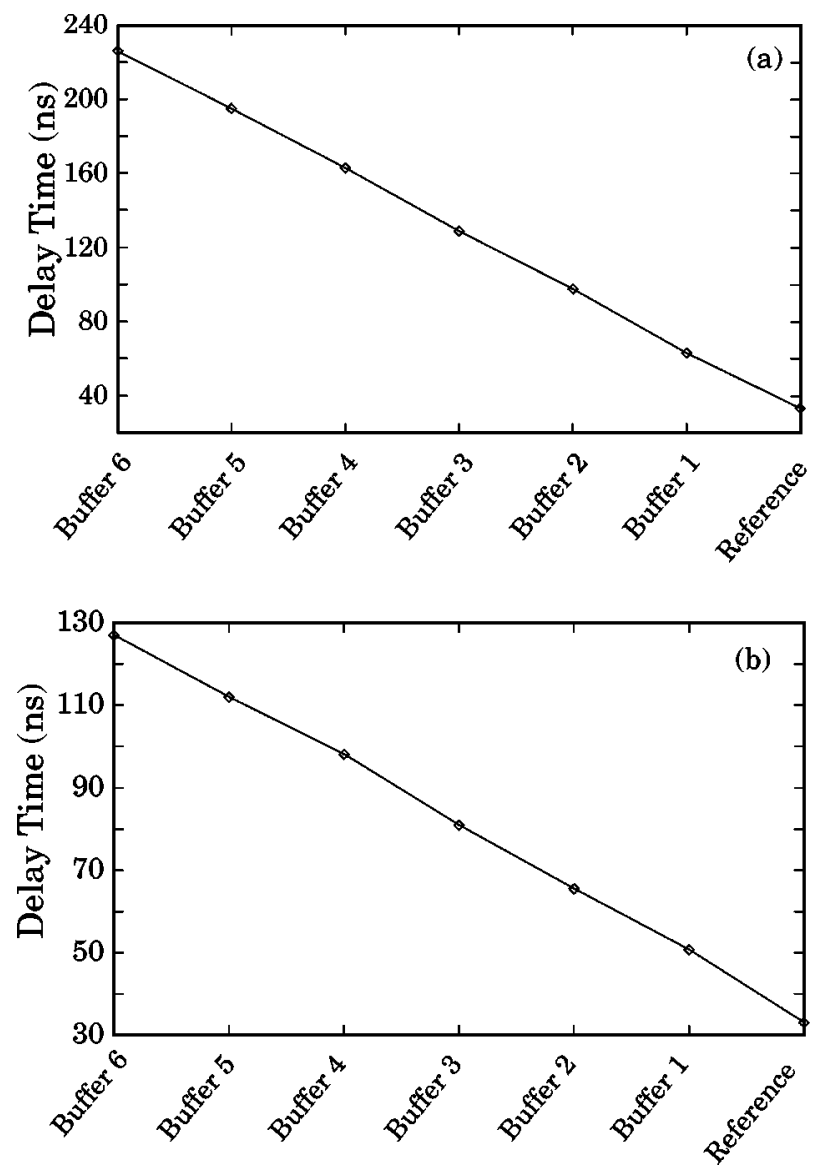

FIG. 6. Propagation time delays of wave forms acquired using: (a) Tektronix TDS520 oscilloscope from all six buffers of the HCF4050UBE noninverting Hex buffer when supplied with $5 \mathrm{~V}$ and (b) Tektronix TDS520 oscilloscope from all six buffers of the HCF4050UBE noninverting Hex buffer when supplied with $10 \mathrm{~V}$.

The time delay at the reference point in each case in Fig. 6 is a measurement of the intrinsic delay within the sensor. In both Figs. 6(a) and 6(b) this is close to $35 \mathrm{~ns}$ and can, if required, be subtracted from the accumulated data to give the true delay time, as is the usual practice when using oscilloscopes for phase measurements. In Fig. 6(a) we show a plot of the individual leading edge times as a function of track number, starting on the left with the reference. Here, the time delay from the first buffer is $63.2 \mathrm{~ns}$ and the total delay from all six buffers is 226 ns. In Fig. 5(b) the measurements of Fig. 5(a) have been repeated but for these the supply on the Hex buffer was set at $10 \mathrm{~V}$ so that the time interval between each pair of pulse edges was halved. As can be seen, the leading edges of the pulses are still perfectly discernable although now closer together. In Fig. 6(b) the leading edge times are again plotted as a function of track number. Here, it can be seen that compared to the data of Fig. 6(a) the linearity is not quite so well preserved. In this example the measured time delay from the first buffer is $50.7 \mathrm{~ns}$ and the total propagation delay from all six buffers is $127 \mathrm{~ns}$. Given these measured times, it is of interest to note that the HCF4050UBE data sheet quotes a propagation time delay for each buffer of between 70 and 140 ns at a supply voltage of $5 \mathrm{~V}$ and between 40 and $80 \mathrm{~ns}$ when this is increased to 10 V. 


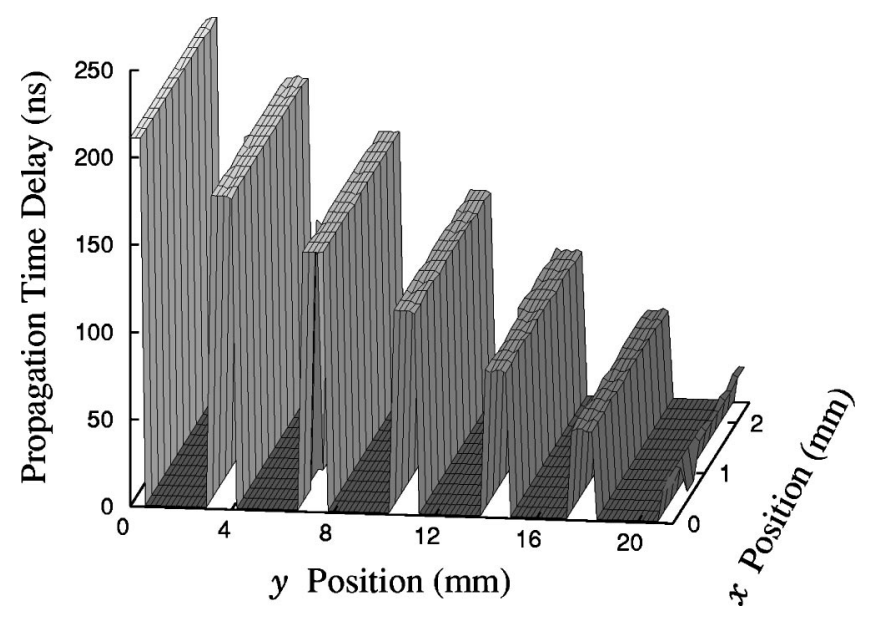

FIG. 7. The propagation time delay from the six buffers of the HCF4050UBE using the PM6680 counter.

In our second approach to measuring propagation time delays across the Hex buffer we made use of the Philips 6680 fast counter (channel A-reference, channel B-signal pickup). For this we set the system to make a raster scan covering the whole of the rectangular area outlined in Fig. 4. As for the data of Figs. 5 and 6, the height of the probe above the Hex buffer PCB was set at $15 \mu \mathrm{m}$ with a scan repeat interval between measurements of $0.2 \mathrm{~mm}$. In Fig. 7, with a supply voltage of $5 \mathrm{~V}$ and a $1 \mathrm{MHz}$ pulse for the reference, we show the two-dimensional image created using the data collected as a result of this raster scan. As can be seen, the image consists of a series of constant time delay mesas, one for each buffer stage. The constant height of each mesa arises simply because of insufficient time resolution along individual tracks in the PCB mounted Hex buffer. With track lengths $\approx 1 \mathrm{~cm}$ concomitant propagation times are $\lesssim_{0.1} \mathrm{~ns}$, beyond the time resolution available to us. However, it is clear that the raster scan staircase image of Fig. 7 does identify in a straightforward and totally unambiguous way each time delay buffer stage in the Hex buffer configuration. From the data displayed in Fig. 7 we find that the propagation time delay from the first buffer stage is $58 \mathrm{~ns}$ and the total delay time for all six buffers is close to $220 \mathrm{~ns}$. These results are very similar to those obtained for the Hex buffer using the oscilloscope display method.

\section{B. Example 2: The PIC16F84-04/P CMOS microcontroller circuit}

As a second example of a digital pulse circuit we decided to look at a microcontroller-the PIC16F84-04/ ${ }^{16}$-as

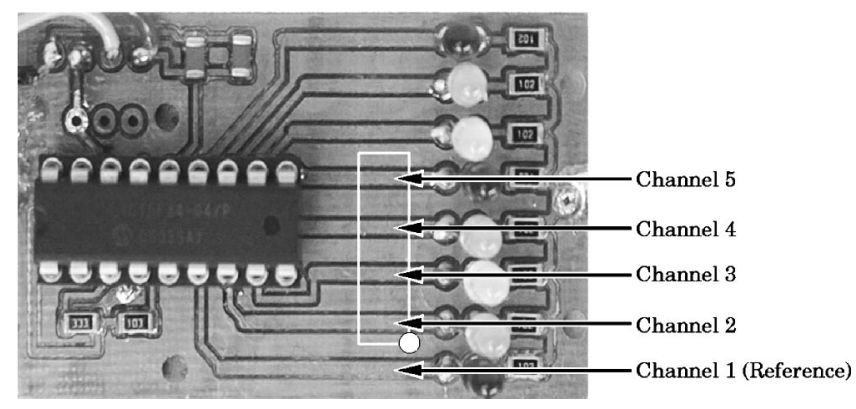

FIG. 8. Photograph of the PIC16F84-04/P microcontroller circuit.

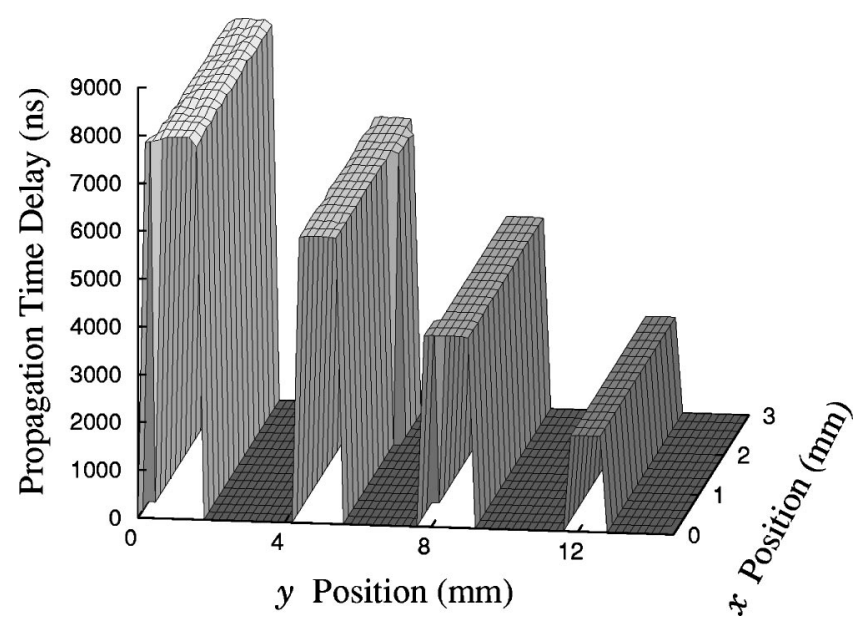

FIG. 9. Detection of the program step duration of a PIC16F84 at an operating frequency of $4 \mathrm{MHz}$.

shown in the photograph of Fig. 8. This is a low-cost, high performance, CMOS 8-bit IC, which employs an advanced RISC architecture with eight output channels. We made this choice to see if the scanning sensor system could be used to detect the program step duration of a PIC microcontroller at different operating frequencies and, as for Fig. 7, the fast counter method was used to create a time delay image in the $x-y$ plane. In operation the PIC microcontroller was programmed with a simple program which just turned on eight light emitting diodes (LEDs) in a sequence, then turned all of the LEDs off and repeated (or looped through) the same sequence again indefinitely.

We set the scanning probe system to cover the rectangular area $(\Delta y=14.6 \mathrm{~mm}$ by $\Delta x=3 \mathrm{~mm})$ marked out in Fig. 8 where, as in Fig. 4, the start of the scan is denoted by a circle. As before, the sensor probe was adjusted to be at 15 $\mu \mathrm{m}$ above the microcontroller board and the step interval (both $x$ and $y$ ) between probe measurements was set at 0.2 $\mathrm{mm}$. The reference for the Philips counter (to channel A) was provided by the output from the PIC microcontroller first channel. We then made a raster scan across the other seven output channels of the PIC (i.e., channels 2-8). In Figs. 9, 10 , and 11 we show time delay images, across channels $2-5$

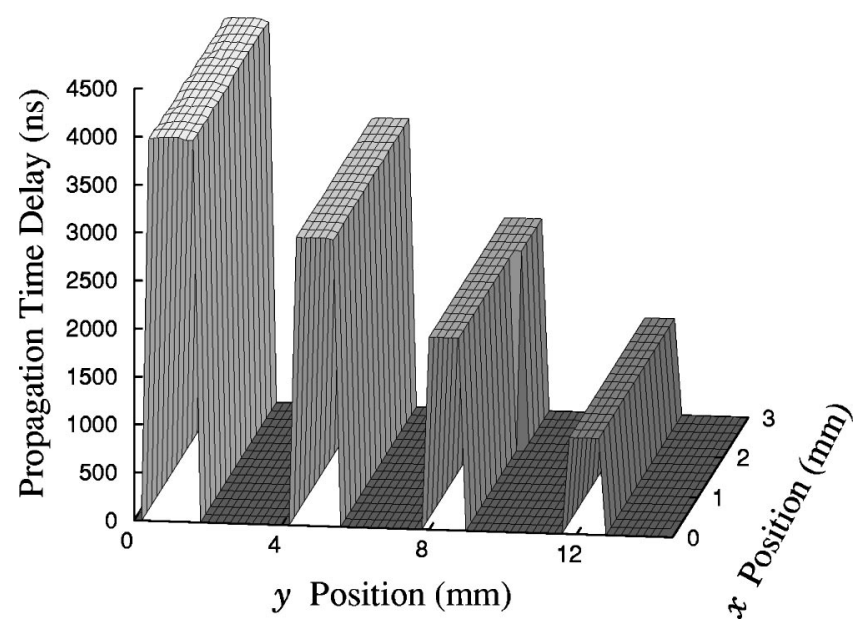

FIG. 10. Detection of the program step duration of a PIC16F84 at an operating frequency of $8 \mathrm{MHz}$. 


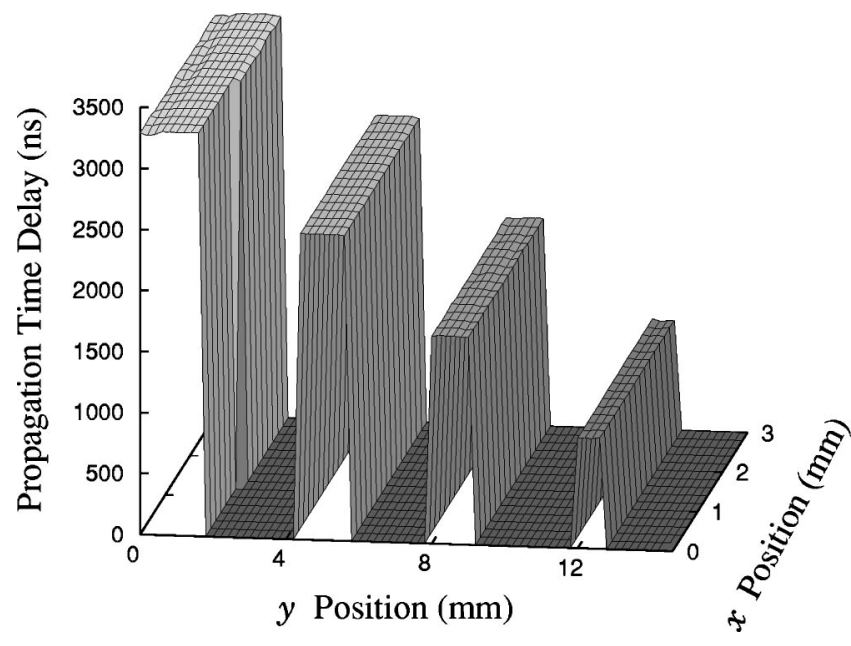

FIG. 11. Detection of the program step duration of a PIC16F84 at an operating frequency of $10 \mathrm{MHz}$.

inclusively, acquired by detecting the program step duration of the PIC16F84 at operating frequencies of 4,8 , and 10 $\mathrm{MHz}$, respectively. The propagation delay at $4 \mathrm{MHz}$ of the PIC second output channel, referred to its first output channel, was $2 \mu$ s. This delay decreased to $1 \mu$ s at an operating frequency of $8 \mathrm{MHz}$ and to $0.8 \mu$ s at $10 \mathrm{MHz}$, i.e., as the PIC gets faster it takes less time for it to execute each of the instructions. Consider, for example, the situation for the 4 $\mathrm{MHz}$ operating frequency case. With a $4 \mathrm{MHz}$ clock $Q 1$ $=($ Clock $/ 4)=1 \mathrm{MHz}$, the time delay $(1 /$ Frequency $)$ $=1 / 1 \mathrm{MHz}=1 \mu$ s., i.e., the PIC microcontroller takes $1 \mu \mathrm{s}$ to execute every instruction, since there were two instructions to step between every two output channels of the PIC $(=2 \times 1 \mu \mathrm{s}=2 \mu \mathrm{s})$. This is the program step duration taken by the PIC to light the LED of channel 2 after channel 1 .

Table II illustrates the results of Figs. 9, 10, and 11. Again it is clear how well the scanning probe imaging technique works in measuring and displaying the propagation delays in this microcontroller circuit.

\section{DISCUSSION}

In this article we have demonstrated that we can apply the technology of ultrahigh input impedance electric potential probes to the measurement of propagation time delays (and imaging) of digital pulse circuits. We have provided two examples: first, a noninverting Hex buffer and, second, a

TABLE II. Time taken by PIC to step between channels at different frequencies.

\begin{tabular}{lccc}
\hline \hline Channels & $4 \mathrm{MHz}$ & $8 \mathrm{MHz}$ & $10 \mathrm{MHz}$ \\
\hline $1-2$ & $2 \mu \mathrm{s}$ & $1 \mu \mathrm{s}$ & $0.8 \mu \mathrm{s}$ \\
$1-3$ & $4 \mu \mathrm{s}$ & $2 \mu \mathrm{s}$ & $1.6 \mu \mathrm{s}$ \\
$1-4$ & $6 \mu \mathrm{s}$ & $3 \mu \mathrm{s}$ & $2.4 \mu \mathrm{s}$ \\
$1-5$ & $8 \mu \mathrm{s}$ & $4 \mu \mathrm{s}$ & $3.2 \mu \mathrm{s}$ \\
\hline \hline
\end{tabular}

microcontroller. We have shown that our present sensor probe circuitry is adequate to deal with pulse repetition frequencies of up to $10 \mathrm{MHz}$ (rise time resolutions of around 15 ns), sufficient to prove the efficacy of the technique. In due course we anticipate that it will prove possible to use this noninvasive technique to monitor pulses with repetition frequencies in the hundreds of $\mathrm{MHz}$ range. In the present work the sensor probes used had a spatial resolution $\approx 0.05 \mathrm{~mm}$, corresponding approximately to the diameter of the probe tip. This resolution was quite adequate to make timing measurements off-chip, on both the surrounding circuit tracks and connection pads. Future reductions in probe dimensions, and improved probe electronics, may allow us to make such measurements (and imaging) on-chip. We note that this has already been achieved at $1 \mu \mathrm{m}$ resolution ${ }^{3}$ with electric potential probes operating in the audio frequency range $(\approx 1$ $\mathrm{kHz}$ ). Ultimately, we may look to planar, two-dimensional arrays of microprobes to generate pulse propagation images of IC digital chips in essentially real time. Such array systems may also find biological application for in vitro (and in saline) imaging of signals between coupled networks of heart or nerve cells.

\section{ACKNOWLEDGMENTS}

The authors would like to thank the Engineering and Physical Sciences Research Council for its generous funding of this research. We would also like to express our gratitude to D. Smith and M. Nock for their technical contributions to the work described in this article.

${ }^{1}$ See, for example, A. J. Clippingdale, R. J. Prance, T. D. Clark, and C. Watkins, Rev. Sci. Instrum. 65, 269 (1994)

${ }^{2}$ A. J. Clippingdale, R. J. Prance, T. D. Clark, and F. Brouers, J. Phys. D 27, 2426 (1994)

${ }^{3}$ R. J. Prance, T. D. Clark, H. Prance, and A. J. Clippingdale, Meas. Sci. Technol. 9, 1229 (1998).

${ }^{4}$ Electron Beam Testing Technology, edited by J. T. L. Thong (Plenum, New York, 1993).

${ }^{5}$ J. Vac. Sci. Technol. 14: Proceedings 8th International Conference on Scanning Tunnelling Microscopy/Spectroscopy and Related Techniques, Colorado, 23-28 July 1995.

${ }^{6}$ D. Hoffman, A. Haas, T. Kunstmann, J. Seifritz, and R. Moller, J. Vac. Sci. Technol. A 15, 1418 (1997).

${ }^{7}$ R. Moller, C. Baur, A. Esslinger, and P. Kurz, J. Vac. Sci. Technol. B 9, 609 (1991).

${ }^{8}$ G. Naubauer and A. Erikson, J. Vac. Sci. Technol. B 14, 426 (1996).

${ }^{9}$ R. J. Prance, A. Debray, T. D. Clark, H. Prance, M. Nock, C. J. Harland, and A. J. Clippingdale, Meas. Sci. Technol. 11, 291 (2000).

${ }^{10}$ OPA 111AM from Burr Brown, 6730S Tucson Boulevard, Tucson, Arizona 85706.

${ }^{11}$ INA 116UA from Burr Brown, 6730S Tucson Boulevard, Tucson, Arizona 85706 .

${ }^{12}$ OPA 655P from Burr Brown, 6730S Tucson Boulevard, Tucson, Arizona 85706.

${ }^{13}$ Ealing Electro-optics PLC, Greycaine Road, Watford WD2 4PW, England.

${ }^{14}$ National Instruments Corp. (UK) Ltd., 21 Kingfisher Court, Hambridge Road, Newbury, Berkshire RG14, United Kingdom.

${ }^{15}$ SGS-Thomson Microelectronics, 165 Rue Edouard Branly, BP 112Technopark du Pays de Gex, F-01637 Saint-Genis-Pouilly Cedex, France.

${ }^{16}$ AZ Microchip Tech. Ltd., Microchip House, 505 Eskdale Road, Wokingham, Berkshire RG41 5TU, England. 